\title{
MAHONY'S INTRIGUING STIFF EQUATIONS
}

\author{
ROBERT E. O'MALLEY, JR. ${ }^{1}$
}

(Received 10 September 1997; revised 11 September 1997)

Professor John Mahony, F.A.A., was a talented and unusual Australian applied mathematician (cf. Fowkes and Silberstein [6]), trained in Manchester in the early 1950s under James Lighthill and Richard Meyer. He may be best remembered today for his early work on multiple scales ([8]), for the soliton equation named after him and his collaborators Brooke Benjamin and Jerry Bona ([2]) and for the many students and colleagues he influenced positively. This note concerns certain illustrative examples listed in the three-part paper Stiff Systems of Ordinary Differential Equations by John and his then postdoc John Shepherd, published in the Journal of the Australian Math. Society (Series B) ([9]). After skimming their eighty-seven pages, it is hard to tell how thoroughly they understood the behavior of solutions to their sample problems (though these descriptions remain the most compelling parts of the papers). I can now admit that, sometime in the late 1970 s, I recommended that (perhaps an early version of some of) these papers not be published in (I think) a SIAM journal. I am now glad Series B accepted them. Indeed, with regard to Mahony ([8]), Fowkes and Silberstein ([6]) reported "It is likely, in fact, that the JAMS paper was rejected by other more prestigious journals. This was often the case with John's work; partially because his material was almost always a departure from conventional wisdom, but also because John's writing could be rather formal and obscure." The junior author of the 1981 papers now has achieved considerable mastery of the subject area, but couldn't have been expected to then take the helm from the opinionated Mahony who had initiated the study through his successful proposal to the Australian Research Council.

Stiff equations occur as numerically-challenging initial value problems for systems of ordinary differential equations. Due to the work of Bill Gear, Germund Dahlquist, Larry Shampine and other numerical analysts in the 1970s, sophisticated software to solve them is now routinely available. The close (but still partially veiled) relationship between singular perturbations and stiffness has long been of mutual interest to the asymptotics and numerics communities (e.g., $[1,7]$ and $[10]$ ). In particular, those of

\footnotetext{
'Department of Applied Mathematics, Box 352420, University of Washington, Seattle, Washington 98195, USA.

(C) Australian Mathematical Society 1999, Serial-fee code 0334-2700/99
} 
us aiming to develop asymptotic approximations of solutions need to use stiff codes to see what happens as a critical positive parameter ( $\epsilon$, say) tends to a limiting value (like zero). Likewise, asymptotic solutions for stiff problems can provide valuable insight to those designing efficient computational algorithms to overcome stiffness.

When I discussed such matters with Michael Osborne of the Australian National University in the (northern) summer of 1996, he suggested that I look at Mahony's papers with both Shepherd and John Norbury. When I did, I remembered that I had long ago vowed to analytically figure out how the solutions to their problems behaved. My favorite examples were $\epsilon \dot{y}=t\left(1-y^{2}\right)$ and $\epsilon \dot{y}=t\left(g^{2}(t)-y^{2}\right)$ on $t \geq-1$ for $g(t)>0$ and $\dot{g}(t)>0$. The first problem is separable and has the exact solution

$$
y_{\epsilon}(t)=\frac{(y(-1)+1)+(y(-1)-1) e^{\left(1-t^{2}\right) / \epsilon}}{(y(-1)+1)-(y(-1)-1) e^{\left(1-t^{2}\right) / \epsilon}} .
$$

For $y(-1)<1$ and $y(-1) \neq-1$, it features an $O(\epsilon)$-thick region of non-uniform convergence abutting $t=-1$ and has the limiting solution -1 for $|t|<1$ with a sudden jump to 1 occurring in an $O(\epsilon)$ neighborhood of $t=1$ if $y(-1)>-1$ and to $-\infty$ if $y(-1)<-1$. Since the limit -1 is linearly unstable on $0<t<1$, the delayed jump is an example of the relatively rare phenomenon of a canard ([3]). For the second problem, however, when $-g(-1)<y(-1)<g(-1)$, the second jump from $-g(t)$ to $g(t)$ occurs in a thicker $O(\sqrt{\epsilon})$ neighborhood of $t=0$ (as Leonid Kalachev and I worked out by matching or "patching").

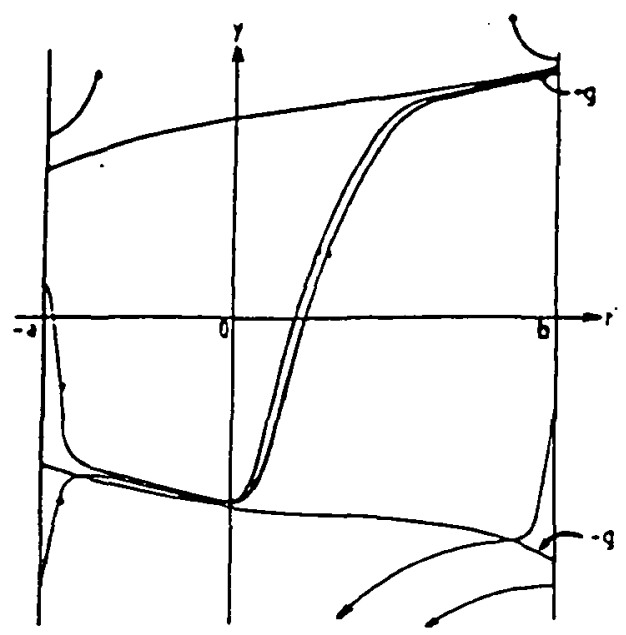

FIGURE 1 .

That's not obvious, however, from the sketch provided by Mahony and Shepherd (reproduced as Figure 1). Indeed, the qualitative value of their figures is problematic 
since, as they said, "in the figures we have not used very large slopes to indicate boundary or interior layers." The obvious question is where the shock is located when $g(t)=1+\alpha t$ for some positive, but asymptotically small, $\alpha$. As the clever authors knew, "the system is then able to remember exponentially small terms, and these control the position of interior layers."

Let me limit attention to the first equation considered by Mahony and Shepherd; namely $\epsilon \dot{y}=t^{2}\left(t^{2}-y^{2}\right)$ on $t \geq-1$ (cf. their Figure 2). Leonid Kalachev and I used MATLAB to plot solutions for $\epsilon=10^{-2}$ and $10^{-4}$ and $y(-1)=0$ (cf. Figures 3 and 4).

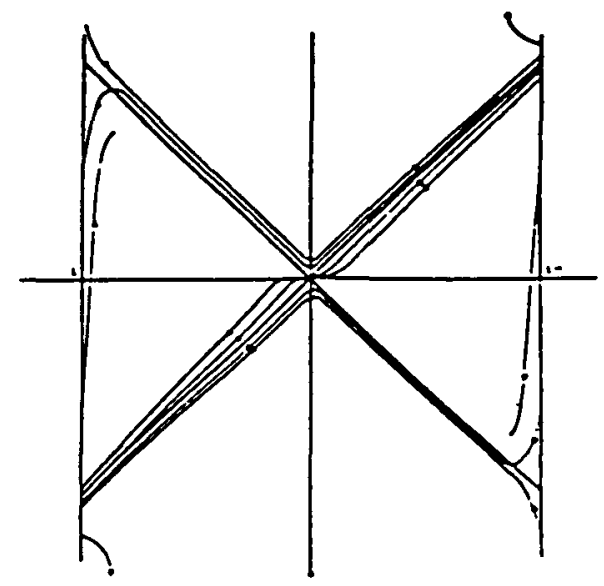

FIGURE 2.

We found an initial layer of $O(\epsilon)$ thickness near $t=-1$ and an outer limit $|t|$ for $|t|<1$, except for some smearing of the anticipated corner layer at $t=0$, where the appropriate outer limit must switch from $-t$ to $+t$. Indeed, a breakdown when $y=O\left(\epsilon^{1 / 4}\right)$ and $t=O\left(\epsilon^{1 / 4}\right)$ must be expected since, for example, the left outer expansion $Y_{L}(t, \epsilon)=-t-\frac{1}{2} \epsilon t^{-3}+\frac{7}{8} \epsilon^{2} t^{-7}+\ldots$ becomes disordered there as its terms attain comparable sizes. As a (singularly perturbed) Riccati equation, it's natural (though highly nontrivial) to convert the given ODE to a linear second-order equation which can be classically solved in terms of the modified Bessel functions $I_{3 / 8}\left(t^{4} / 4 \epsilon\right)$ and $K_{3 / 8}\left(t^{4} / 4 \epsilon\right)$ and their first derivatives (see [5] or [12]). Imagine my surprise when Grant Keady pulled me to his office (after l'd given a talk in Perth) to show me how a student version of MAPLE immediately provided the solution

$$
y_{\epsilon}(t)=-t\left[\frac{K_{-5 / 8}\left(t^{4} / 4 \epsilon\right)-C_{L} I_{-5 / 8}\left(t^{4} / 4 \epsilon\right)}{K_{3 / 8}\left(t^{4} / 4 \epsilon\right)+C_{L} I_{3 / 8}\left(t^{4} / 4 \epsilon\right)}\right]
$$




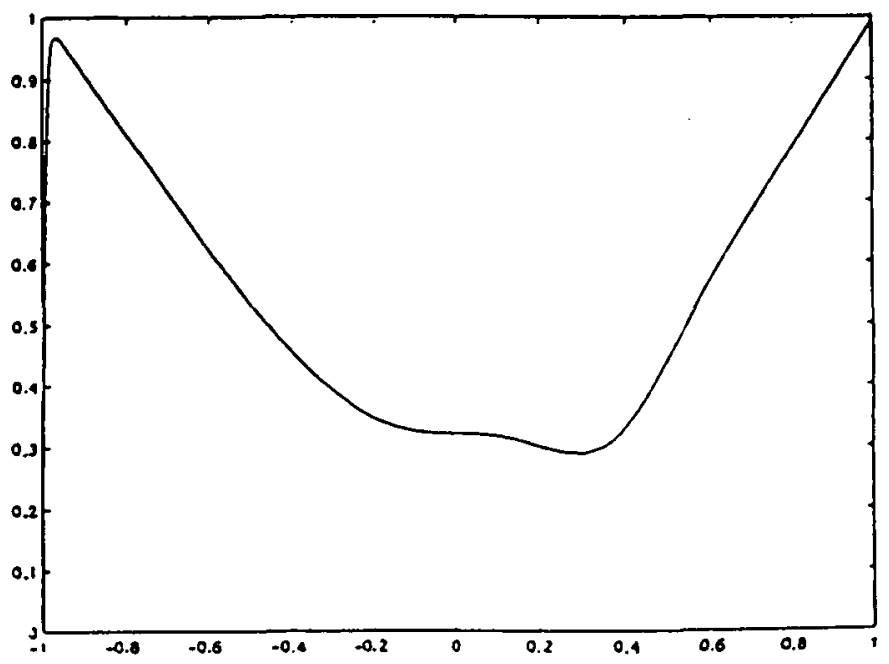

FIGURE 3.

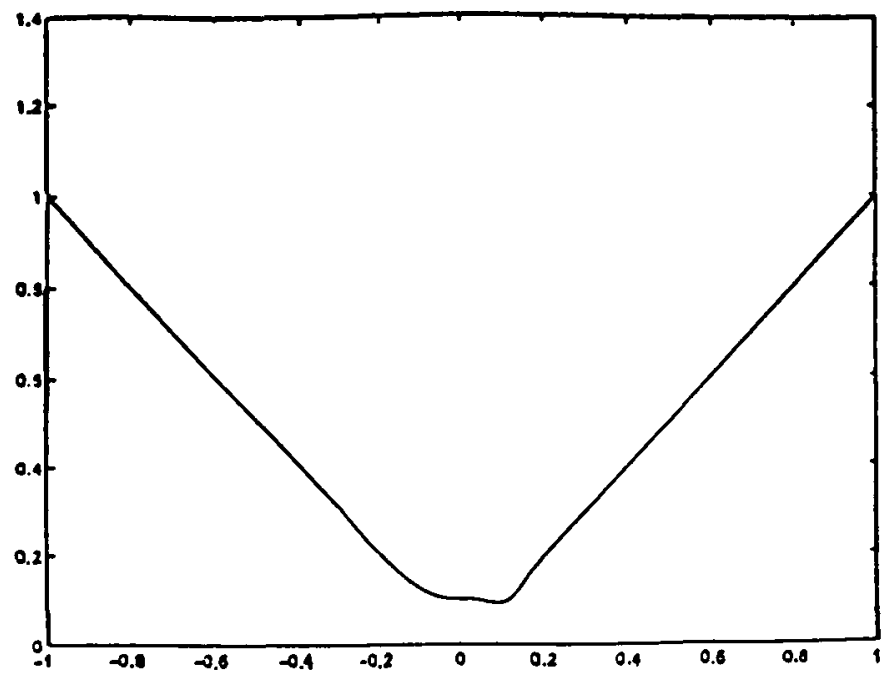

FIGURE 4. 
for a constant $C_{L}$ ! The initial value $y(-1)$ clearly determines a unique $O\left(e^{-\frac{1}{2 \epsilon}}\right)$ value $C_{L}$, provided $y(-1)>-1$. Asymptotic approximations for the Bessel functions show the usual $O(\epsilon)$ thick initial layer at $t=-1$ and the outer solution $y_{L}(t, \epsilon)$ for $-1<t<0$. The poles the $K_{v}$ 's and $I_{-5 / 8}$ have at $t=0$ show that $y$ has a removable singularity there with the limit $y_{\epsilon}(0) \sim \Gamma(5 / 8)(8 \epsilon)^{1 / 4} / \Gamma(3 / 8)$ for all allowed initial values. Since $\dot{y}_{\epsilon}(0)=0$, the jump in the MAPLE plot there was wrong. It missed Mahony and Shepherd's insight that the solution was smooth at the origin. This meant that it should have instead used

$$
y_{\epsilon}(t)=t\left[\frac{C_{R} I_{-5 / 8}\left(t^{4} / 4 \epsilon\right)-K_{-5 / 8}\left(t^{4} / 4 \epsilon\right)}{C_{R} I_{3 / 8}\left(t^{4} / 4 \epsilon\right)+K_{3 / 8}\left(t^{4} / 4 \epsilon\right)}\right]
$$

for $t>0$ with a new constant $C_{R}$. Indeed, matching at $t=0$ forces us to take $C_{R}$ to asymptotically be $\Gamma(3 / 8) \Gamma(5 / 8)$, thereby providing the expected outer solution

$$
Y_{R}(t, \epsilon)=t-\frac{\epsilon}{2} t^{-3}+\ldots
$$

for $t>0$. As the MATLAB solutions showed, the solution isn't even in $t$. Because the $O\left(\epsilon^{1 / 4}\right)$ solution isn't so small near $t=0$, numerical difficulties aren't even severe until $\epsilon$ is really very small. Then, however, one must be careful to make sure the computed solution switches from the limit $-t$ to $+t$ there ( $c f$. the related knee problem of Dahlquist et al. ([4]) noting their concern about numerical superstability).

The vision Mahony and Shepherd had was "to consider properties of solutions of a few simple first-order systems as a guide to what might be expected in higherorder systems." That hope remains today as we finally come to somewhat more fully appreciate the value of the intriguing examples they introduced.

In closing, let me acknowledge the help of numerous friends worldwide, who all continue to find Mahony's problems provocative.

\section{Acknowledgement}

This work was supported in part by the National Science Foundation under grant number DMS 9703382.

\section{References}

[1] R. C. Aiken, Stiff Computation (Oxford University Press, Oxford, 1985).

[2] T. B. Benjamin, J. L. Bona and J. J. Mahony, "Model equations for long waves in non-linear dispersive systems", Phil. Trans. Royal Soc. Ser. A 272 (1972) 47-78. 
[3] E. Benoit (ed.), Dynamic Bifurcations, Lecture Notes in Math. 1493 (Springer-Verlag, Berlin, 1991).

[4] G. Dahlquist, L. Edsberg, G. Skollermo and G. Soderlind, Are the Numerical Methods and Software Satisfactory for Chemical Kinetics?, Lecture Notes in Math. 968 (Springer-Verlag, Berlin, 1982) 149-164.

[5] M. V. Fedoryuk, Asymptotic Analysis (Springer-Verlag, Berlin, 1993).

[6] N. Fowkes and J. P. O. Silberstein, "John Mahony, 1929-1992", Historical Records of Australian Science 10 (1995) 265-291.

[7] E. Hairer and G. Wanner, Solving Ordinary Differential Equations II. Stiff and DifferentialAlgebraic Problems (Springer-Verlag, Berlin, 1991).

[8] J. J. Mahony, "An expansion method for singular perturbation problems", J. Aust. Math. Soc. 2 (1962) 440-463.

[9] J. J. Mahony and J. J. Shepherd, "Stiff systems of ordinary differential equations, Parts I, II, and III", J. Austral. Math. Soc. (Series B) 23 (1981) 17-51, 136-172, and 310-331.

[10] W. L. Miranker, Numerical Methods for Stiff Equations (Reidel, Dordrecht, 1981).

[11] R. E. O'Malley, Jr., "Stiff differential equations and singular perturbations", Research report, Centre for Mathematics and its Applications, Australian National University, 1996.

[12] A. D. Polyanin and V. F. Zaitsev, Handbook of Exact Solutions for Ordinary Differential Equations (CRC Press, Boca Raton, 1995). 